\title{
Chitosan/polyester-based scaffolds for cartilage tissue engineering: Assessment of extracellular matrix formation
}

\author{
M.L. Alves da Silva ${ }^{\mathrm{a}, \mathrm{b}, \mathrm{d}, *}$, A. Crawford ${ }^{\mathrm{d}}$, J.M. Mundy ${ }^{\mathrm{d}}$, V.M. Correlo ${ }^{\mathrm{a}, \mathrm{b}}$, P. Sol ${ }^{\mathrm{a}, \mathrm{b}}$, M. Bhattacharya ${ }^{\mathrm{c}}$, \\ P.V. Hatton ${ }^{\mathrm{d}}$, R.L. Reis ${ }^{\mathrm{a}, \mathrm{b}}$, N.M. Neves ${ }^{\mathrm{a}, \mathrm{b}}$ \\ a 3B's Research Group, Biomaterials, Biodegradables and Biomimetics, University of Minho, Headquarters of the European Institute of Excellence on Tissue Engineering \\ and Regenerative Medicine, AvePark, 4806-909 Caldas das Taipas, Guimarães, Portugal \\ ${ }^{\mathrm{b}}$ IBB, Institute for Biotechnology and Bioengineering, PT Associated Laboratory, Guimarães, Portugal \\ ${ }^{\mathrm{c}}$ Department of Biosystems Engineering, University of Minnesota, USA \\ ${ }^{\mathrm{d}}$ Centre for Biomaterials and Tissue Engineering, University of Sheffield, Sheffield, UK
}

\section{A R T I C L E I N F O}

\section{Article history:}

Received 6 April 2009

Received in revised form 21 August 2009

Accepted 15 September 2009

Available online 27 September 2009

\section{Keywords:}

Cartilage tissue engineering

Chitin/chitosan

Bovine chondrocyte

Scaffold

\begin{abstract}
A B S T R A C T
Naturally derived polymers have been extensively used in scaffold production for cartilage tissue engineering. The present work aims to evaluate and characterize extracellular matrix (ECM) formation in two types of chitosan-based scaffolds, using bovine articular chondrocytes (BACs). The influence of these scaffolds' porosity, as well as pore size and geometry, on the formation of cartilagineous tissue was studied. The effect of stirred conditions on ECM formation was also assessed. Chitosan-poly(butylene succinate) (CPBS) scaffolds were produced by compression moulding and salt leaching, using a blend of $50 \%$ of each material. Different porosities and pore size structures were obtained. BACs were seeded onto CPBS scaffolds using spinner flasks. Constructs were then transferred to the incubator, where half were cultured under stirred conditions, and the other half under static conditions for 4 weeks. Constructs were characterized by scanning electron microscopy, histology procedures, immunolocalization of collagen type I and collagen type II, and dimethylmethylene blue assay for glycosaminoglycan (GAG) quantification. Both materials showed good affinity for cell attachment. Cells colonized the entire scaffolds and were able to produce ECM. Large pores with random geometry improved proteoglycans and collagen type II production. However, that structure has the opposite effect on GAG production. Stirred culture conditions indicate enhancement of GAG production in both types of scaffold.
\end{abstract}

( 2009 Acta Materialia Inc. Published by Elsevier Ltd. All rights reserved.

\section{Introduction}

There are four major types of cartilage, which can be distinguished by their specific constitutive components: hyaline cartilage, fibrocartilage, elastic cartilage and costochondral cartilage [1]. Hyaline articular cartilage is the most abundant type in the body, composed of one cell type (the chondrocyte) dispersed in an abundant extracellular matrix (ECM). ECM is composed mainly of collagen type II and a large proteoglycan, aggrecan $[1,2]$. The ECM provides most of the functional properties associated with hyaline cartilage, including resistance to compression and provision of low friction articulating surfaces in the joints. Injuries to cartilage are often painful and may severely affect movement.

\footnotetext{
* Corresponding author. Address: 3B's Research Group, Biomaterials, Biodegradables and Biomimetics, Department of Polymer Engineering, University of Minho, Headquarters of the European Institute of Excellence on Tissue Engineering and Regenerative Medicine, AvePark, Zona Industrial da Gandra, S. Cláudio do Barco, 4806-909 Caldas das Taipas, Guimarães, Portugal. Tel.: +351 253 510900; fax: +351 253510909.

E-mail address: msilva@dep.uminho.pt (M.L. Alves da Silva).
}

Unfortunately, articular cartilage has a relatively poor capacity for self-repair (related to the lack of a direct blood supply), and cartilage injury is frequently associated with the onset of chronic problems, including osteoarthritis $[1,3]$.

Currently, there is no agreed method of restoring fully damaged cartilage [4]. Current therapies include abrasion arthroplasty, subchondral drilling, prosthetic joint replacement and, ultimately, transplantation of autologous chondrocytes or tissues [4,5]. However, these treatments do not constitute a complete recovery for the patient and, in most cases, persistent problems of donor site morbidity, limitations of patient mobility and consequent disability, loss of implants and limited durability of the prosthetics [2,4-6] are observed.

Tissue engineering represents a promising approach for the repair of articular cartilage, but there is still no ideal scaffold for this approach. Chitosan/polyesters appear to offer several advantages in this field.

In recent years, natural-based polymers, such as, for example, chitosan, have been studied extensively [7-11]. Chitosan is a derivative of chitin, obtained by deacetylation of this biopolymer [12]. 
Chitin is the second most abundant polysaccharide in nature, being found usually in crustaceans' shells. Chitosan is a linear polysaccharide with a structure similar to glycosaminoglycans (GAGs) present in native cartilage ECM [13]. This property is extremely important for cartilage tissue engineering, since it allows the development of several types of scaffold. Another interesting property of chitosan is that it can be moulded into various shapes [14] and allows for the formation of different pore sizes structures [12]. It has an intrinsic antibacterial activity and high biocompatibility [13]. Chitosan has been used in several blends to produce many types of scaffold, e.g. hydrogels [15,16], porous scaffolds [11], amino acid immobilization [9] or drug delivery [17]. The authors' group has developed innovative blends of synthetic polymers with chitosan $[7,8,18,19]$, which have been developed for biomedical applications such as bone [20-22] or cartilage [23,24]. Studies have been conducted preparing chitosan-based scaffolds aimed at cartilage regeneration, which showed evidence of favourable responses in vitro [20,21,24-26]. As for in vivo studies, a high degree of biocompatibility of chitosan scaffolds has been shown in mice [13]. Another study, conducted in rabbits, observed hyaline-like tissue after 24 weeks of implantation [15]. Recently, the basis for a cartilage engineering model in a large animal has been established using Merino sheep and chitosan-based scaffolds [27]. Using ovine MSC combined with chitosan and TGF- $\beta 3$, histological analysis revealed chondrocyte-like cells surrounded by a hyaline-like cartilaginous matrix that was well integrated with the host cartilage [27]. The bio-functionality of chitosan scaffolds produced by particle aggregation was tested in rats in vivo, which showed promising results in terms of connective tissue in-growth and neo-vascularization [28].

In the current study, a bovine chondrocyte model was used to establish primary cultures, and the formation of ECM in chitosan/polyester-based scaffolds was evaluated. Two types of scaffolds with different pore sizes and pore geometries were studied, and ECM deposition on both types of scaffolds was evaluated. In addition, different culture conditions were used, namely static vs. dynamic, in order to establish the most suitable method for obtaining hyaline cartilage tissue in vitro.

\section{Materials and methods}

\subsection{Scaffolds production}

A chitosan-poly(butylene succinate) (CPBS) (50/50 wt.\%) blend was produced and processed into scaffolds using a methodology based on compression moulding followed by salt leaching, as previously described $[8,18]$. Common salt $(\mathrm{NaCl})$ was used as the porogen agent. Two different groups of scaffolds were produced: one using 80 wt.\% salt with particles size between 63 and $125 \mu \mathrm{m}$ (80 CPBS). The second group of scaffolds was produced using $60 \mathrm{wt} . \%$ salt with particle size between 250 and $500 \mu \mathrm{m}$ (60 CPBS). In both cases, salt and blend were loaded into a mould, heated and compression-moulded into large discs. Discs were sliced to obtain $5 \mathrm{~mm}$ cubes. These cubes were immersed in distilled water to leach out the salt over 6 days. Water was changed every day to facilitate the complete dissolution of the porogen agent. At the end of the procedure, the cubes were dried until a constant weight was obtained. The resulting scaffolds were characterized, and will be referred to as $80 \%$ porosity or $60 \%$ porosity.

\subsection{Isolation of bovine articular chondrocytes (BACS)}

Isolation of BACs was performed according to a method previously reported [29]. Full thickness hyaline cartilage was harvested from bovine metacarpophalangeal joint. The skin was removed, and the joint was transferred to a laminar air hood. Cartilage was dissected in small full-depth pieces and washed twice with PBS buffer (Sigma D-8537). Then it was digested with $0.25 \%(\mathrm{w} / \mathrm{v}$ ) of trypsin solution (Sigma E-5134) for $30 \mathrm{~min}$ at $37^{\circ} \mathrm{C}$ on a rotator. The solution was removed, cartilage was washed again in PBS buffer and then incubated in a collagenase type I solution $\left(2 \mathrm{mg} \mathrm{ml}^{-1}\right.$ ) (Sigma E0130) overnight at $37^{\circ} \mathrm{C}$ on a rotator (Stuart mini orbital shaker SSM1). The following day, cells were washed twice with PBS, counted and plated at a density of $2 \times 10^{6}$ cells per Petri dish. Cells were cultivated with expansion medium: Dulbecco's modified Eagle's medium (DMEM; high glucose) (Sigma D-5671), containing $10 \mathrm{mM}$ Hepes buffer (Sigma H-0887), l-alanyl-l-glutamine (Sigma G-8541), non-essential aminoacids (Sigma M-7145) 10,000 units $\mathrm{ml}^{-1}$ penicillin, $10,000 \mu \mathrm{g} \mathrm{ml}^{-1}$ streptomycin (Sigma P-0781), $10 \%$ foetal calf serum (Biosera S1810) and $10 \mathrm{ng} \mathrm{ml}^{-1}$ basic fibroblast growth factor (bFGF) (PeproTech 100-18B). The culture medium was changed twice a week, and cells were expanded until passage 2 .

\subsection{Bovine articular chondrocyte (BAC) culture on chitosan-based scaffolds}

BAC seeding was performed dynamically in spinner flasks. Cells were harvested, counted and resuspended in expansion medium. Twenty scaffolds were used in each experiment, for each type of scaffold. Materials were seeded with a cell suspension containing $6.5 \times 10^{5}$ cells per scaffold. Seeding was performed in spinner flasks, using a magnetic stirrer placed inside an incubator at $37^{\circ} \mathrm{C}$ in order to allow cell penetration into the material's porous structure. After cell seeding was complete, constructs were removed from spinner flasks. Two experimental conditions were set: dynamic and static. For dynamic culture conditions, half the constructs were incubated at $37^{\circ} \mathrm{C}$ on a rotator at $60 \mathrm{rpm}$. For static conditions, constructs were left to culture statically inside the incubator. The culture medium was changed every 3 days. At this stage, the culture medium used was differentiation medium (expansion medium without bFGF and with $1 \mathrm{mg} \mathrm{ml}^{-1}$ of insulin (Sigma Co.) and $1 \mathrm{mg} \mathrm{ml}^{-1}$ of ascorbic acid) (Sigma A-4544). Samples were taken at different time points: 1, 2, 3 and 4 weeks of culture.

\subsection{Microcomputed tomography $(\mu \mathrm{CT})$}

Microcomputed tomography $(\mu \mathrm{CT})$ equipment (SkyScan, Belgium) was used as a non-destructive technique for very detailed analysis of the morphology of the developed scaffolds. Four scaffolds of each condition were scanned in high-resolution mode of $8.7 \mu \mathrm{m} x / y / z$ and an exposure time of $1792 \mathrm{~ms}$. The energy parameters defined in the scanner were $63 \mathrm{keV}$ with a current of $157 \mu \mathrm{A}$. Isotropic slice data were obtained by the system and reconstructed into two-dimensional (2D) images. These slice images were compiled and analysed to render three-dimensional (3D) images and obtain quantitative architecture parameters. A $\mu \mathrm{CT}$ analyser and a $\mu C T$ Volume Realistic 3D Visualization, both from SkyScan, were used as image processing tools both for $\mu \mathrm{CT}$ reconstruction and to create/visualize the 3D representation. Regions of interest $(4.5 \times 4.5 \mathrm{~mm}$ squares $)$ were selected in each slice image, and a threshold was set to eliminate background noise. This threshold (to distinguish polymer material from pore voids) was chosen and maintained constant for all the scanned specimens and samples. The threshold was also inverted to obtain pore volume and to analyse the pore morphology.

\subsection{Scanning electron microscopy}

For scanning electron microscopy (SEM) analysis, constructs were collected at every time point. They were washed in sterile PBS and immersed in 3\% glutaraldehyde (Sigma G-5882) with 
0.1 M cacodylate buffer $\mathrm{pH} 7.4$ (AGAR R1103) at room temperature for $30 \mathrm{~min}$. Afterwards, they were washed in PBS buffer three times to remove all glutaraldehyde from the surface. Then constructs were submerged in osmium tetraoxide and left for $2 \mathrm{~h}$. Finally, they were dehydrated in alcohol and left to air dry. The samples were splutter-coated with gold and analysed by SEM. Two different scanning electron microscopes were used for this work: a Philips XL-20 and a Leica Cambridge S360 (Leica Cambridge, Cambridge, UK).

\subsection{Histological analysis (hematoxylin-eosin, toluidine blue, alcian blue)}

Samples were collected at the end of the experiment ( 4 weeks of culture) and embedded in OCT (OCT compound BDH, Gurr) in order to make them suitable for cryosectioning, and stored at $-20^{\circ} \mathrm{C}$. Sections were cut at $8 \mu \mathrm{m}$ and placed on microscopy slides. A fresh 4\% paraformaldehyde (Sigma P-6148) solution in PBS buffer was prepared, filtered and cooled down for fixation of the slides. Slides were fixed for $30 \mathrm{~min}$ at $4{ }^{\circ} \mathrm{C}$, washed twice in distilled water and left to air dry overnight. Slides were subsequently stored at $4{ }^{\circ} \mathrm{C}$ until used for staining procedures. Hematoxylin and eosin (H\&E) staining was conducted in an automatic machine fume cupboard (X219/E11/LEV1). In this procedure, sections were washed in running tap water for $5 \mathrm{~min}$, and then dipped in $1 \%$ acid alcohol for 5 $10 \mathrm{~s}$. Sections were washed again in tap water and stained in eosin for $10 \mathrm{~min}$. Another wash with tap water was performed for $5 \mathrm{~min}$, and sections were afterwards dehydrated through alcohols. They were cleared in xylene and mounted in DPX (BDH 36029 2F). Toluidine blue staining was performed on a selection of the sections. The staining solution was prepared by dissolving $1 \%$ of toluidine blue (Sigma T-0394) in distilled water containing $0.5 \mathrm{~g}$ of sodium borate, followed by filtering. One drop of this solution was added to each section for 2-3 s. Then, sections were rinsed with distilled water and allowed to air dry overnight. Sections were cleared and mounted as described previously. Alcian blue staining was performed by rinsing the sections in 3\% acetic acid and incubating them in $1 \%$ alcian blue solution (Sigma A-3157) for $18 \mathrm{~h}$. After that, the stain was poured off, and sections were counterstained with aqueous neutral red (Sigma N-6634) for $1 \mathrm{~min}$. Sections were washed with water, left to air dry and then rinsed in absolute alcohol, cleared and mounted as described previously.

\subsection{Immunolocalization of type I and II collagens}

Immunolocalization of type I and type II collagens was performed in fixed sections. Sections were washed in PBS and pretreated with $10 \mathrm{mg} \mathrm{ml}^{-1}$ hyaluronidase (Sigma Co.) in PBS for $30 \mathrm{~min}$ at $37^{\circ} \mathrm{C}$ and with $2 \mathrm{mg} \mathrm{ml}^{-1}$ pronase (Fluka/Sigma Co.) again for $30 \mathrm{~min}$ at $37^{\circ} \mathrm{C}$. Then, sections were washed in PBS, and endogenous peroxidase activity was quenched with $3 \%$ hydrogen peroxide in 50\% methanol (BDH 101586 6B) for 5 min. Sections were washed in Tri-buffered saline (TBS) solution and blocked with 3\% BSA (Sigma A-2153) in TBS/Tween 20 (Sigma Ultra P-7949) for $1 \mathrm{~h}$ to avoid non-specific staining. Sections were then incubated with primary antibodies (collagen type I and collagen type II) (goat anti-type I collagen UNLB 1310-01 and goat anti-type II collagen UNLB 1320-01) overnight at $4{ }^{\circ} \mathrm{C}$ in a humidified atmosphere. The next day, sections were washed once with high salt wash solution and twice in TBS/Tween 20 for 10 min each and then incubated with secondary antibody from the kit for $1 \mathrm{~h}$ at room temperature, again in a humidified atmosphere. The remaining protocol is as described in the Vectastain Elite ABC Kit PK-6105 (Vector Laboratories Ltd., UK) and in the Vector DAB Kit (Vector Laboratories Ltd., UK). Slides were washed in water for $5 \mathrm{~min}$ and then counterstained with haematoxylin for nuclei visualization. Then slides were mounted in DPX. Controls were performed using normal goat serum instead of primary antibodies, which was also included in the kit.

\subsection{Dimethylmethylene blue assay for glycosaminoglycan (GAG) quantification}

For this assay samples were collected at 4 weeks of culture, freeze/dried overnight and then digested. This digestion allowed the separation from the scaffold of the ECM formed. Digestion solution was prepared by adding papain (Sigma P-4762) and $N$-acetyl cysteíne (Sigma A-8199), to obtain the final concentrations of $0.05 \%$ and $0.096 \%$, respectively, to $50 \mathrm{ml}$ of digestion buffer (200 mM of phosphate buffer containing $1 \mathrm{mM}$ EDTA (Sigma E5134), pH 6.8). Samples were placed in $1.5 \mathrm{ml}$ tubes and incubated with $600 \mu \mathrm{l}$ of the relevant solution, overnight at $60^{\circ} \mathrm{C}$. Afterwards, samples were centrifuged in a bench centrifuge at $13,000 \mathrm{rpm}$ for 10 min. Supernatant was collected and stored at $-20^{\circ} \mathrm{C}$ until the GAG assay was performed. Solutions for this assay were prepared as follows. Dimethylmethylene blue (DMB) stock solution was prepared by dissolving $16 \mathrm{mg}$ DMB powder in $900 \mathrm{ml}$ of distilled water containing $3.04 \mathrm{~g}$ glycine and $2.73 \mathrm{~g} \mathrm{NaCl}$. This was mixed for $2 \mathrm{~h}$, covered with aluminium foil. $\mathrm{pH}$ was adjusted to 3.0 with $\mathrm{HCl}$ and volume to $1 \mathrm{l}$. The solution was stored at room temperature covered with aluminium foil. Chondroitin sulphate (Sigma C-8529) solution was prepared in water, in a $5 \mathrm{mg} \mathrm{ml}^{-1}$ stock solution and kept refrigerated. This solution was diluted with water, resulting in 5 unit increments from 0 to $50 \mu \mathrm{g} \mathrm{ml}^{-1}$, in order to make a standard curve. Samples were also diluted as appropriate with distilled water. To a 96 well plate, $20 \mu \mathrm{l}$ of water was added as a blank. The same quantity of chondroitin sulphate diluted solution was added in duplicate, and the same was performed with all the samples. To each well, $250 \mu \mathrm{l}$ of DMB solution was added in a multichannel pipette, and the optical density was measured in a microplate reader at $530 \mathrm{~nm}$. Scaffolds without seeded cells were used as controls.

\subsection{Reproducibility of the experiments}

Each scaffold seeding was performed three times with cells isolated from a different animal.

\subsection{Statistical analysis}

Data from triplicates of GAG quantification are present as average \pm standard errors. One-way analysis of variance in conjunction with Tukey's test was performed on the GAG assay results.

\section{Results}

2D and 3D $\mu \mathrm{CT}$ images from both groups of scaffolds show differences between their morphology (Figs. 1 and 2). Scaffolds of 60 CPBS (Fig. 1A) show fewer pores than 80 CPBS scaffolds (Fig. 1B), as expected. Scaffolds of 60 CPBS presented smaller pores. Correlo et al. recently produced and characterized these two types of scaffolds in terms of porosity, pore size and mechanical properties [18]. The 80 CPBS scaffolds were shown to have $78.6 \pm 2.5 \%$ porosity, and a pore size of $276.8 \pm 52.5 \mu \mathrm{m}$. However, 60 CPBS scaffolds were shown to have lower porosity ( $57.7 \pm 6.6 \%)$, as well as a smaller pore size $(199.3 \pm 5.3 \mu \mathrm{m})$ [18].

Pore geometry was also different in both compositions. As can be observed in the 2D $\mu \mathrm{CT}$ images, pores of the 60 CPBS scaffolds have a geometrically defined shape (similar to the salt structure), and seem to be homogeneously distributed throughout the scaf- 

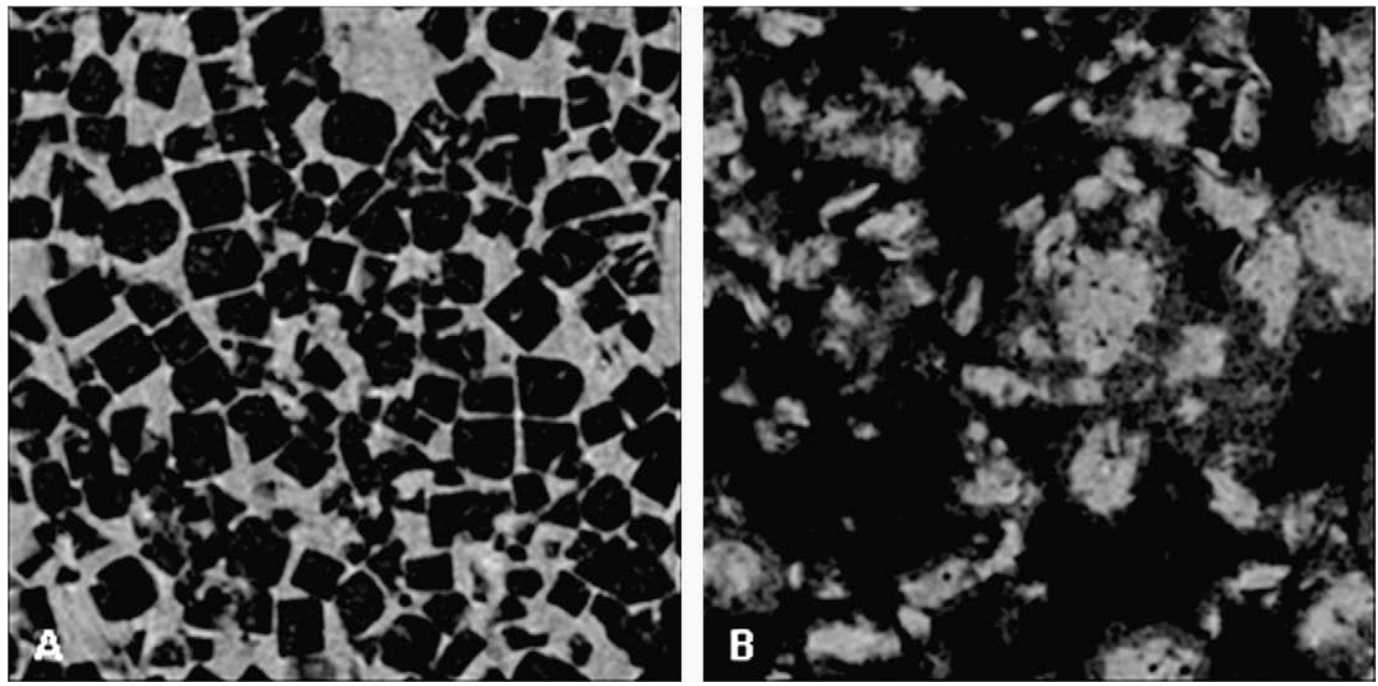

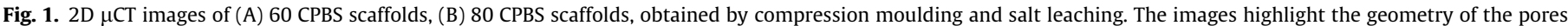
obtained.
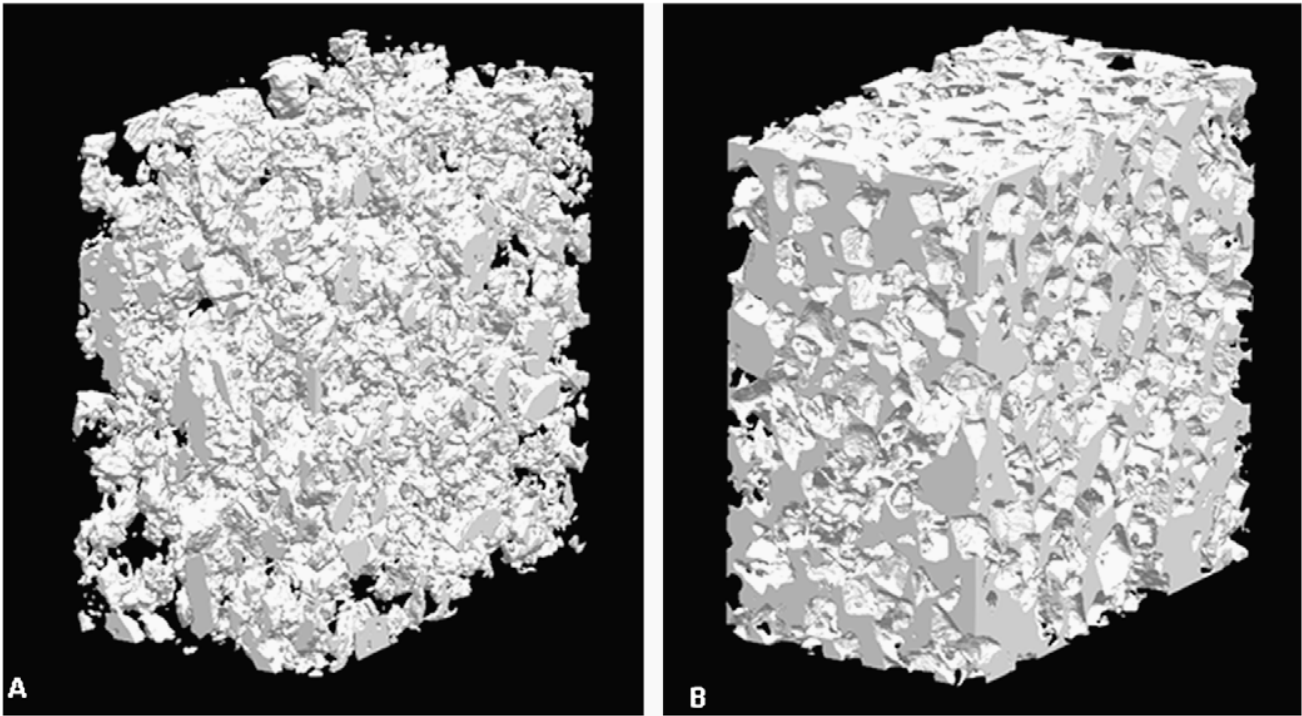

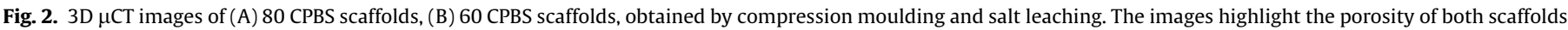

folds. In contrast, the pores of the 80 CPBS scaffolds are randomly distributed and do not present a specific shape.

The SEM micrographs presented in Fig. 3 show the distribution of cells in the seeded scaffolds throughout the period of the experiment. It can be observed that the chondrocytes colonized the entire scaffold in both types of material. Cells kept their roundshaped morphology, typical of chondrocytes, in both static culture (Fig. 3A-D; I-L) and stirred culture conditions (Fig. 3E-H; M-P). Nevertheless, it was observed that the colonization of 60 CPBS scaffolds appeared slower than in 80 CPBS scaffolds in both culture conditions.

The colonization of the scaffolds by cells was shown by H\&E staining (Fig. 4A, D, G and J). H\&E staining showed more cells covering the surface of the 80 CPBS scaffolds than in the 60 CPBS ones. Consequently, more proteoglycans were observed, as shown in the results of toluidine blue (Fig. $4 \mathrm{H}$ and $\mathrm{K}$ ) and alcian blue (Fig. $4 \mathrm{I}$ and L) staining. This observation applies for both culture conditions (static and dynamic). It is important to highlight the alcian blue staining results. The staining in the $80 \mathrm{CPBS}$ scaffolds seems to be stronger and again cover more surface area than that obtained for 60 CPBS scaffolds, in both culture conditions. These results indicate a higher concentration of sulphated proteoglycans in these samples.

The results of immunolocalization of collagens type I and type II in both groups of scaffolds are presented in Fig. 5. Cells seeded into 60 CPBS scaffolds produced both collagen type I and collagen type II (Fig. 5A-F), in both static (Fig. 5B and C) and stirred culture conditions (Fig. 5D and F). In contrast, collagen type I was discrete in the section of 80 CPBS scaffolds (Fig. $5 \mathrm{H}$ and $\mathrm{K}$ ), whereas collagen type II synthesis was revealed in all samples (Fig. 5I and L).

GAGs presented in both types of scaffolds were quantified by the DMB assay, and the results are presented in Fig. 6. Significantly higher GAG production in 60 CPBS scaffolds was detected in comparison with 80 CPBS scaffolds. Stirred culture conditions significantly $(P<0.05)$ enhanced GAG production in both types of scaffolds when compared with static culture conditions. There was a statistical difference in GAG production in 60 CPBS scaffolds when cultured in stirred conditions compared with the data ob- 

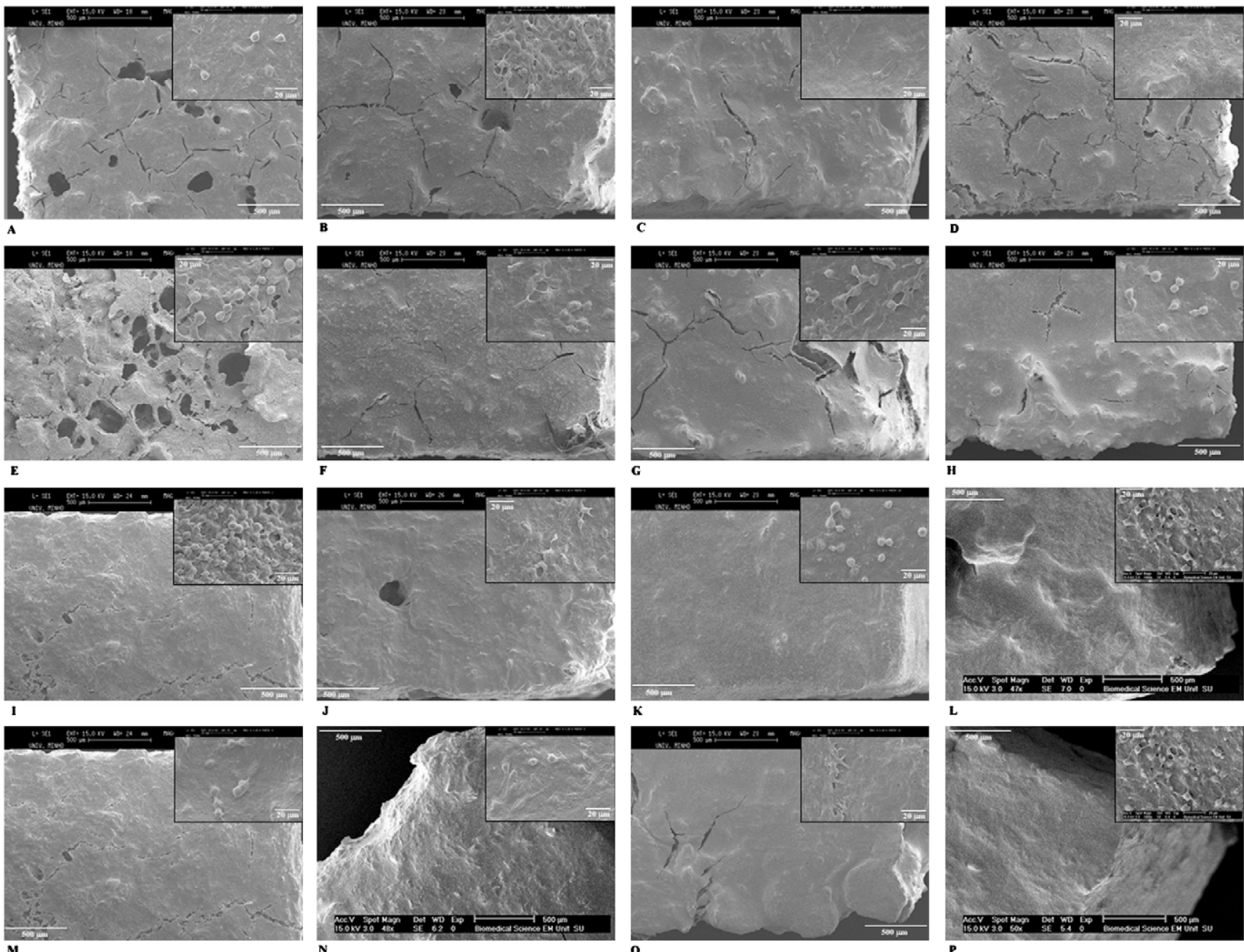

G

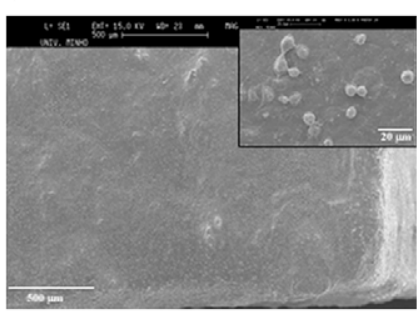

$\mathbf{K}$
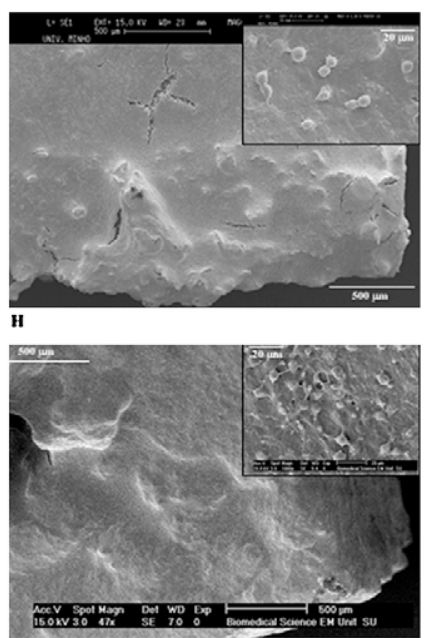

$\mathbf{L}$

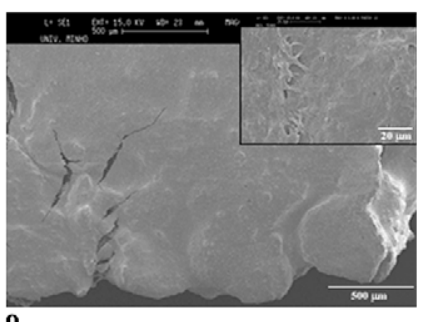

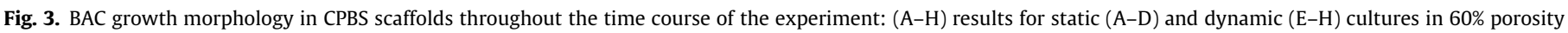
scaffolds; (I-P) results for static (I-L) and dynamic (M-P) cultures in $80 \%$ porosity scaffolds. Different magnifications were used to highlight cell morphology.

tained for static culture conditions. For 80 CPBS scaffolds, GAG production in stirred culture conditions was higher, but not statistically different.

\section{Discussion}

The structure of pores is one important factor in tissue regeneration. The growth of some specific cell types in injured sites is dependent on optimal pore size and geometry [30]. Pore size affects cell response in terms of attachment, growth and proliferation [31]. Variation in pore size also affects the mechanical stability of constructs, and this is an important factor when implanting them in load-bearing areas as well as evaluating the construct response to mechanical stimuli in bioreactors [32]. In particular, permeability affects the shear stresses inside the construct [33], which is a stimulus for cellular differentiation or functional adaptation of the construct during the implant. Furthermore, the mechanical environment in the tissue has a controlling influence on tissue differentiation [33]. For in vivo implantation, cells need to be expanded in vitro to a sufficient number in order to generate a construct with certain mechanical stability [34]. Furthermore, upon implantation, the scaffolds and cells will be subjected to the action of synovial fluid flow in the joint. The fluid flow is believed to facilitate cell migration and promote positive signals by mechanotransduction to the cells. Therefore, the dynamic culture conditions may support high seeding densities and help cells to grow, proliferate and produce ECM. Those conditions are also believed to lead to more stable constructs, and therefore may enhance the construct integration within the tissue at the implant site. In work by Gotterbarm et al. [35], two-layered biomaterials were implanted in osteochondral defects created in the trochlear groove of Göttinger minipigs. They observed cellular migration and cell attachment, as well as matrix production. These authors claim that this cellular filling and attachment was fostered by the porous character of the biomaterials, and led to an increased amount of repaired tissue [35].

In previous work, Correlo et al. produced and characterized several scaffolds produced from different blends of chitosan and synthetic polyesters, including the CPBS scaffolds studied in the present work [18]. A higher range of different types of scaffolds were produced by compression moulding and salt leaching, using different salt sizes to induce small and large pores. Curiously, small salt sizes originated larger pores, owing to the aggregation of the salt particles [18]. The scaffolds from the blend CPBS (50\%/50\%) were chosen based on cytotoxicity and direct contact tests previously performed (data not shown). It was intended to determine the effect of the pore size and shape on the production of ECM by BAC.

In work by Spiteri et al., it was shown that substrate porosity enhanced BAC attachment, growth and formation of cartilage 


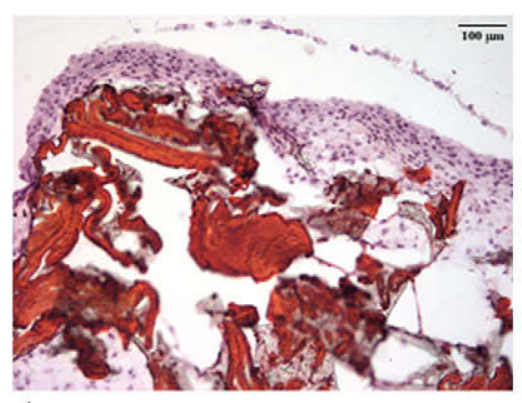

A

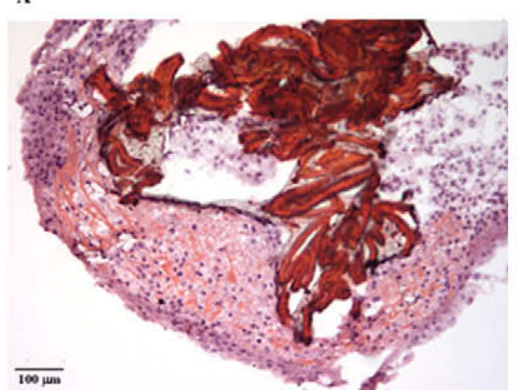

D

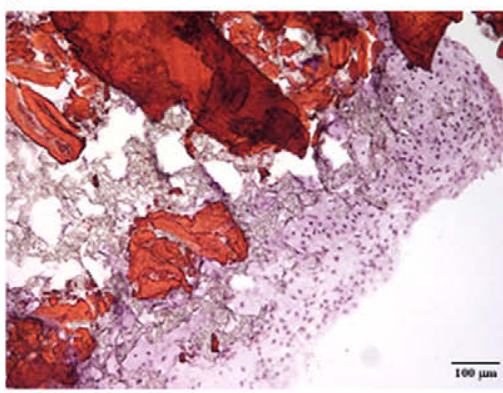

G

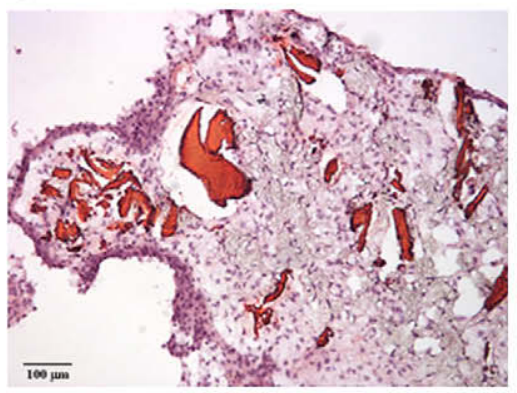

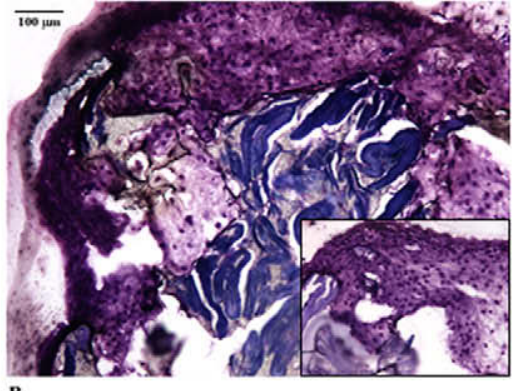

B
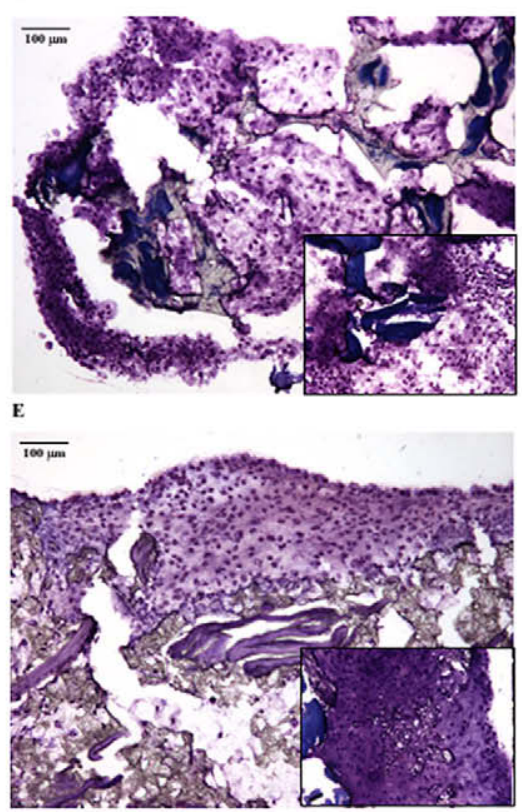

H

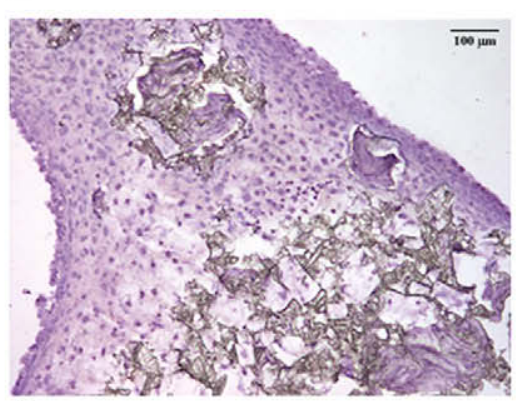

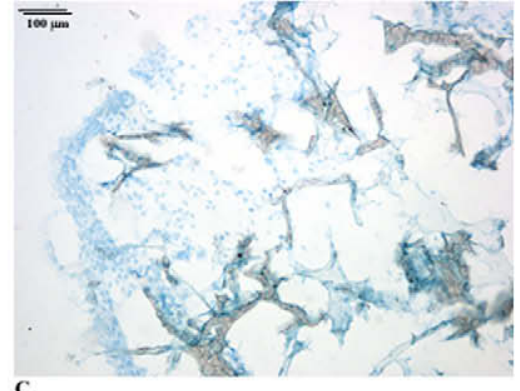

C
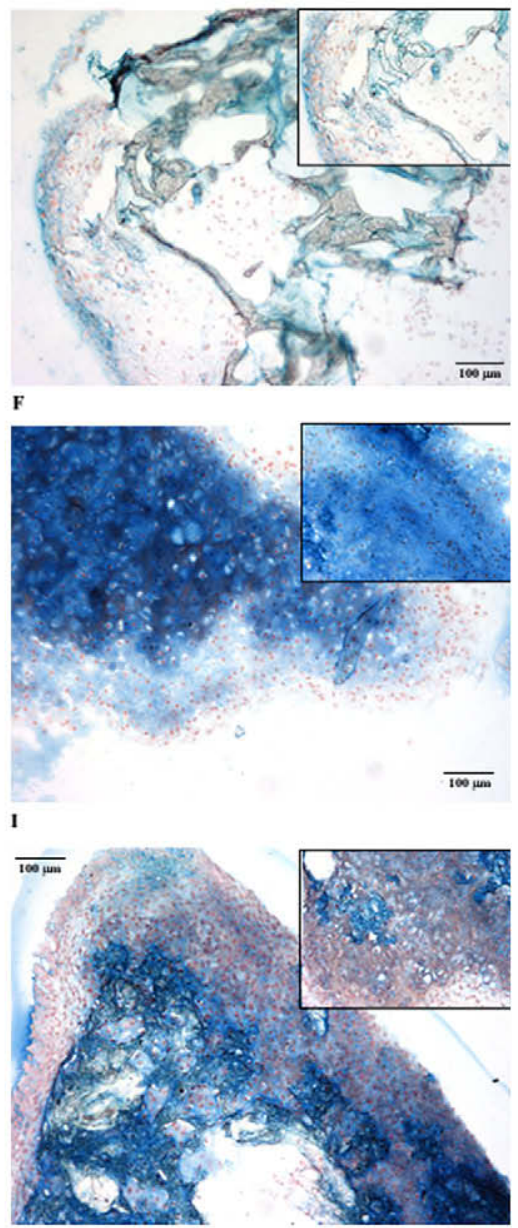

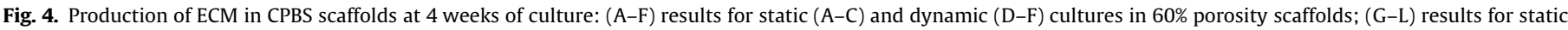

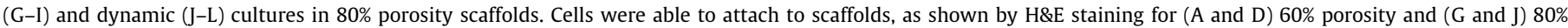

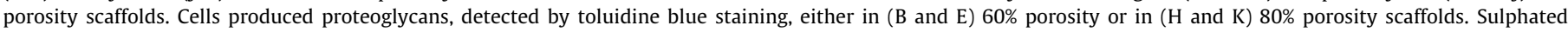

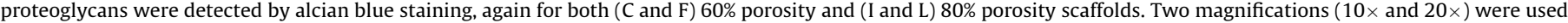
in each staining for microscopic observation. Scale bar $=100 \mu \mathrm{m}$.

in vitro [31]. In the present work, scaffold pore morphology seems to affect BAC attachment and colonization equally. Globally, it is observed that cells attached and colonized both scaffolds. This observation is similar to that obtained when rabbit chondrocytes were seeded onto chitosan-based hyaluronic acid hybrid polymer fibers. In that study, cell proliferation had no significant difference between three groups of scaffolds with different pore sizes [26].

Some studies have focused on the effect of pore size on chondrocyte performance. Cartilaginous tissue obtained on porous titanium alloy discs with the smaller pore size was thicker and had more proteoglycans in comparison with tissue obtained from discs with larger pore sizes [36]. Another study by Nehrer et al. showed that biosynthetic activity and chondrocyte phenotype were im- proved in collagen matrices with smaller pores [37]. It has been suggested that cell-cell interactions are enhanced in scaffolds with small pore sizes, thus resulting in improved chondrocyte proliferation [37]. These studies lead to the conclusion that small pores improve chondrogenesis. However, several other studies point in the opposite direction. Griffon et al. determined that large interconnective pores improve the cellularity and matrix content within chitosan scaffolds [38]. In recent work, Lien et al. also showed that articular chondrocytes of Wistar rats performed better in the group of scaffolds with pore sizes between 250 and $500 \mu \mathrm{m}$ in terms of proliferation and ECM production [39]. In fact, the present findings support these observations. Cells seeded into 60 CPBS produced both collagen type I and collagen type II (Fig. 5A-F), indicative of 

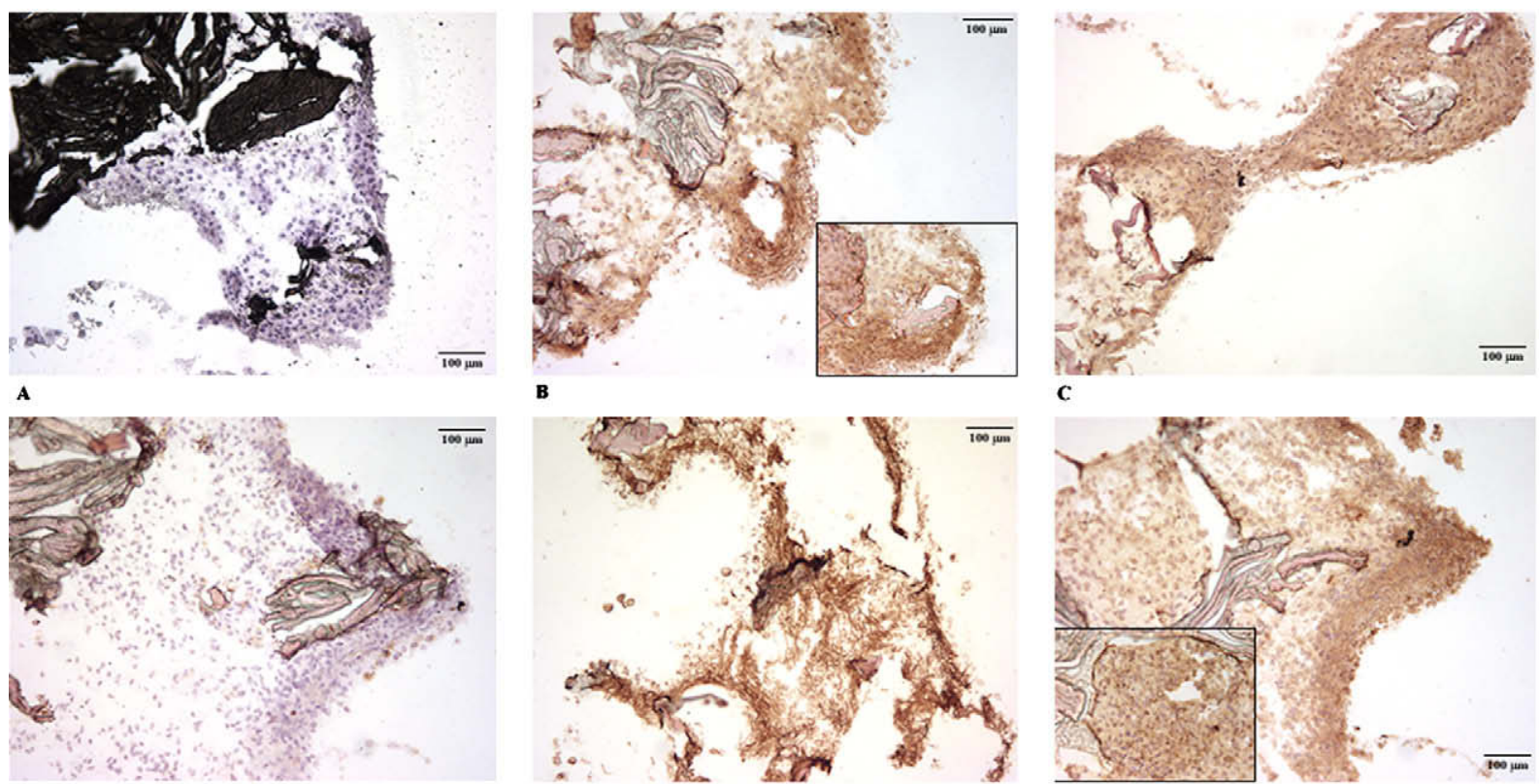

B
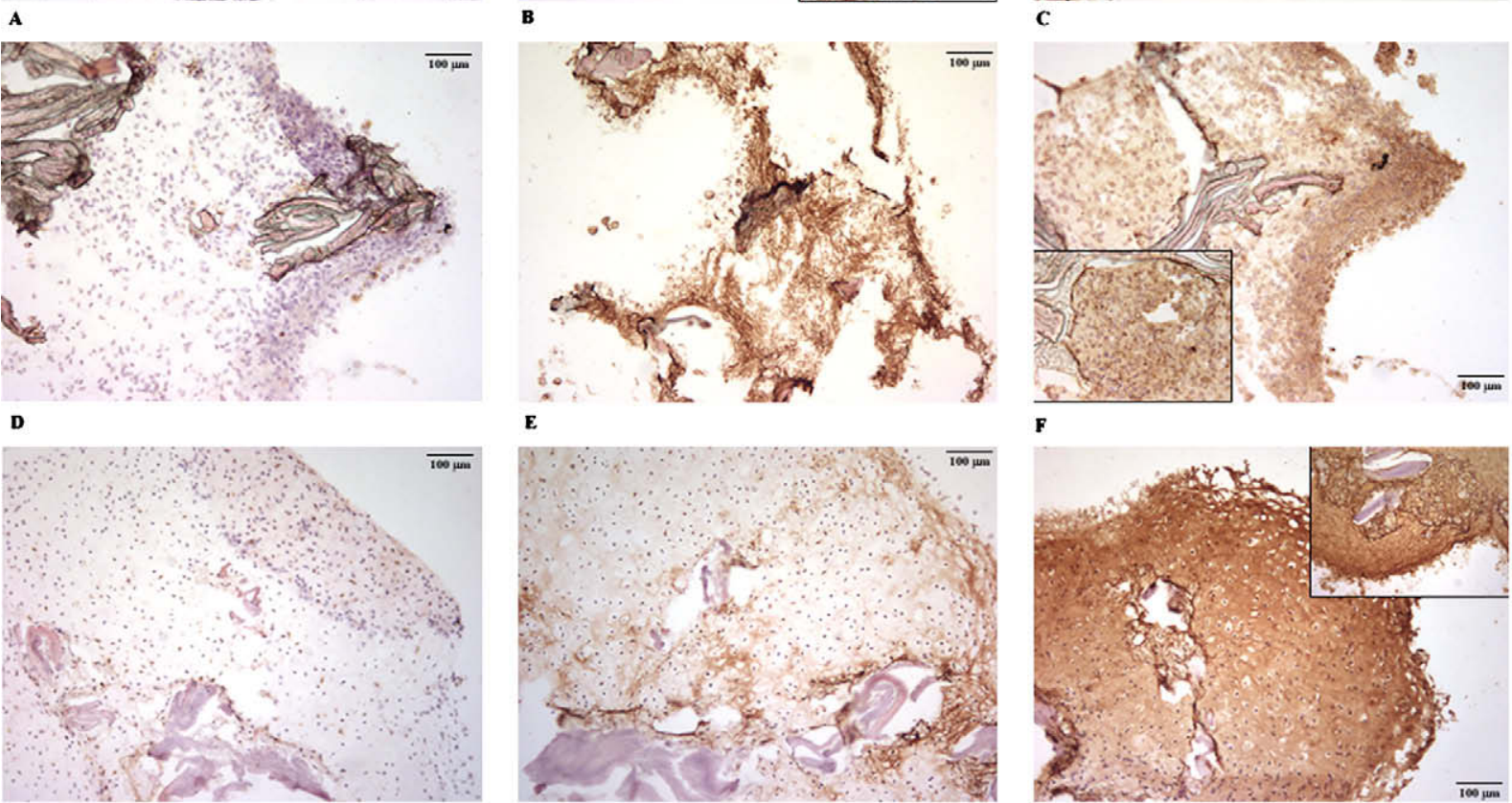

$\mathbf{E}$
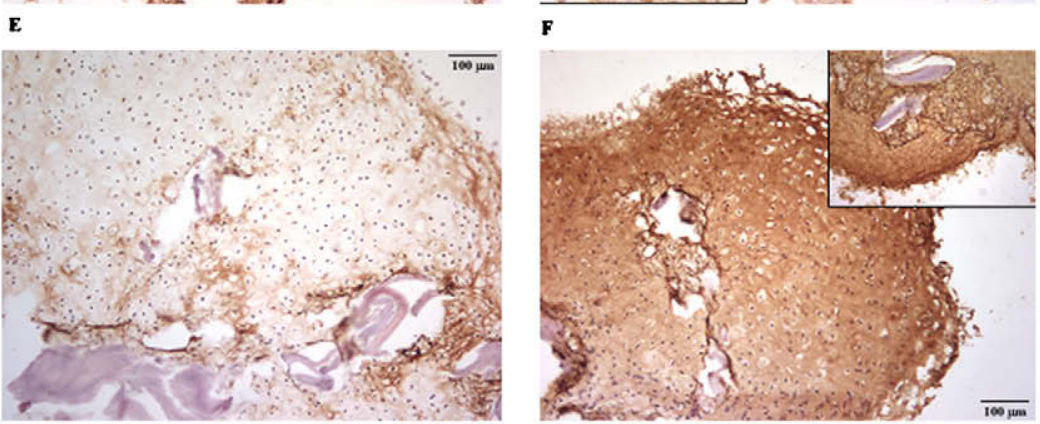

H

I
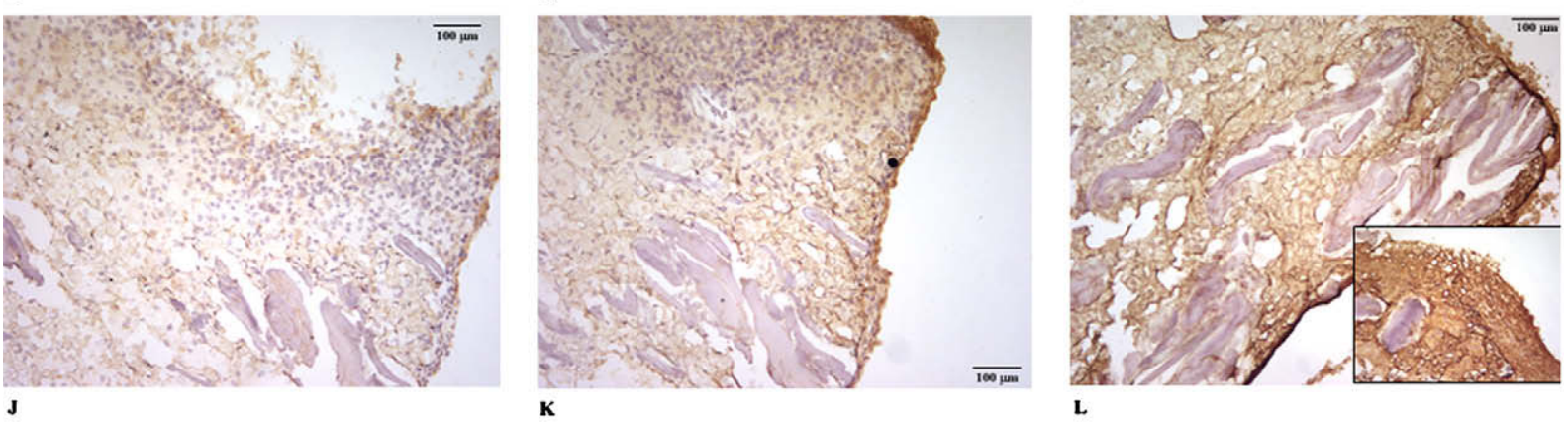

L.

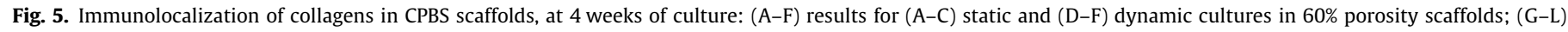

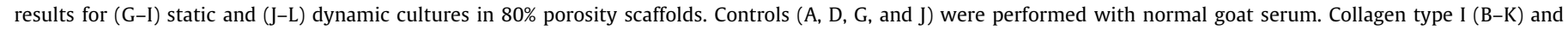
collagen type II $(\mathrm{C}-\mathrm{L})$ were detected. Two magnifications $(10 \times$ and $20 \times)$ were used in each staining for microscopic observation. Scale bar $=100 \mu \mathrm{m}$.

a fibrous cartilage tissue, in either static (Fig. 5B and C) or stirred culture conditions (Fig. 5E and F). Fibrocartilage is a mixed matrix consisting of fibrous tissue and hyaline cartilage. It contains type I and type II collagen and aggrecan [40]. However, hyaline cartilage contains type II collagens and proteoglycans, the major one being aggrecan molecule [40]. Cartilage-like tissue formed in 80 CPBS scaffolds did not presented significant staining of collagen type I (Fig. 5H and $\mathrm{K}$ ) yet showed marked staining for collagen type II, indicating that these tissue properties are more similar to hyaline-like cartilage tissue, than those obtained with 60 CPBS scaffolds. However, GAG content present in 80 CPBS scaffolds was lower compared with that obtained for 60 CPBS scaffolds (Fig. 6). This result seems to contradict the previous ones, going in the direction of the first series of results, where smaller pores enhance proteoglycan deposition [36,37]. In fact, the opposite effect of pore size was observed in this work. On the one hand, large pores induced cell proliferation, production of proteoglycans and collagens. On the other hand, GAG production was significantly lower in scaffolds with large pore sizes. Additionally, stirred culture conditions seem also to affect GAG production for both types of scaffold. In general, it was observed that stirred conditions enhance GAG production. A similar observation was reported by Freyria et al., using 3D collagen scaffolds seeded with BAC and cultured under static or stirred conditions. Thus, authors state that these conditions allow a homogeneous distribution of cells and ECM within scaffolds structure to be obtained [41]. It is described in the literature that substrate geometry and porosity may both influence chondrocyte behaviour $[36,37,42]$. Understanding the impact of biomaterial 


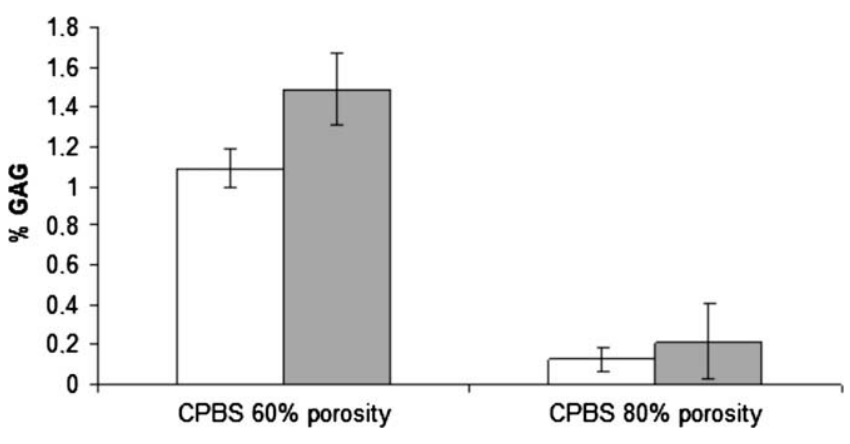

Fig. 6. GAG quantification for CPBS scaffolds at 4 weeks of culture. Static culture conditions are represented in white, dynamic culture conditions in grey.

geometry is very important for enhancing the in vivo ECM formation. Many factors may act in concert, such as fluid flow to enhance nutrition and waste product removal, as well as number of attached cells and their ability to produce ECM [31]. Therefore, the optimal conditions for each scaffold must be determined, because each scaffold's characteristics determine the cell attachment, growth, maintenance of phenotype and ECM production [34]. Relevant characteristics include scaffold texture, porosity (size, structure, distribution) and surface free energy [34]. In summary, every parameter by itself is important and should be considered, but the overall nature of the relevant parameters makes the scaffold functional and suitable for cartilage tissue engineering.

\section{Conclusion}

CPBS scaffolds were produced by compression moulding followed by salt leaching. Two different types of $\mathrm{NaCl}$ salt particles were used as porogens, and two types of scaffolds with different porosities, pore geometry and size were produced. The 80 CPBS scaffolds presented larger, randomly structured pores, in contrast to the 60 CPBS scaffolds, which showed smaller pores with a cubic structure. From the present observations, one can state that 80 CPBS scaffolds seem more effective in inducing ECM production by BACs. The pore size and geometry of the pore had an effect on cell proliferation and ECM production. Proteoglycans and collagen type II were detected in larger quantities in 80 CPBS scaffolds (large pores, random structure) compared with 60 CPBS scaffolds (small pores, cubic structure). Nevertheless, the amount of glycosaminoglycan was lower in the 80 CPBS scaffolds. Large pores affected GAG production in either static or dynamic culture conditions. As regards the culture conditions, stirred conditions improved ECM production in both types of scaffold, thus being preferable to static culture conditions.

\section{Acknowledgements}

M.L. Alves da Silva would like to acknowledge the Portuguese Foundation for Science and Technology (FCT) for her grant (SFRH/ BD/28708/2006), Marie Curie Actions-ALEA JACTA EST (MESTCT-2004-008104), European NoE EXPERTISSUES (NMP3-CT-2004500283), IP GENOSTEM (LSHB-CT-2003-503161) and CARTISCAFF (POCTI/SAU/BMA/58982).

\section{Appendix A. Figures with essential colour discrimination}

Certain figures in this article, particularly Figures 4 and 5, are difficult to interpret in black and white. The full colour images can be found in the on-line version, at doi: 10.1016/ j.actbio.2009.09.006.

\section{References}

[1] Bonassar LJ. Cartilage reconstruction. In: Atala A, Lanza R, editors. Methods of tissue engineering. San Diego: Academic Press; 2002. p. 1027-39.

[2] Nesic D, Whiteside R, Brittberg M, Wendt D, Martin I, Mainil-Varlet P. Cartilage tissue engineering for degenerative joint disease. Adv Drug Deliv Rev 2006;58(2):300-22.

[3] Magne D, Vinatier C, Julien M, Weiss P, Guicheux J. Mesenchymal stem cell therapy to rebuild cartilage. Trends Mol Med 2005;11(11):519-26.

[4] Steinert AF, Ghivizzani SC, Rethwilm A, Tuan RS, Evans CH, Noth U. Major biological obstacles for persistent cell-based regeneration of articular cartilage. Arthritis Res Ther 2007;9(3):213

[5] Martin I, Miot S, Barbero A, Jakob M, Wendt D. Osteochondral tissue engineering. J Biomech 2007;40(4):750-65.

[6] Goessler UR, Hormann K, Riedel F. Tissue engineering with chondrocytes and function of the extracellular matrix (Review). Int J Mol Med 2004;13(4): 505-13.

[7] Correlo VM, Boesel LM, Bhattacharya M, Mano JF, Neves NM, Reis RL. Hydroxyapatite reinforced chitosan and polyester blends for biomedical applications. Macromol Mater Eng 2005;290:1157-65.

[8] Correlo VM, Boesel LM, Bhattacharya M, Mano JF, Neves NM, Reis RL Properties of melt processed chitosan and aliphatic polyester blends. Mater Sci Eng A 2005;403:57-68.

[9] Ho M-H, Wang D-M, Hsieh H-J, Liu H-C, Hsien T-Y, Lai J-Y, et al. Preparation and characterization of RGD-immobilized chitosan scaffolds. Biomaterials 2005;26:3197-206.

[10] Medrado GCB, Machado CB, Valerio P, Sanches MD, Goes AM. The effect of a chitosan-gelatin matrix and dexamethasone on the behavior of rabbit mesenchymal stem cells. Biomed Mater 2006;1:155-61.

[11] Zhao L, Chang J. Preparation and characterization of macroporous chitosan/ wollastonite composite scaffolds for tissue engineering. J Mater Sci Mater Med 2004;15(5):625-9.

[12] Di Martino A, Sittinger M, Risbud MV. Chitosan: a versatile biopolymer for orthopaedic tissue-engineering. Biomaterials 2005;26:5983-90.

[13] VandeVord PJ, Matthew HW, DeSilva SP, Mayton L, Wu B, Wooley PH. Evaluation of the biocompatibility of a chitosan scaffold in mice. J Biomed Mater Res 2002;59(3):585-90.

[14] Hu Q, Li B, Wang M, Shen J. Preparation and characterization of biodegradable chitosan/hydroxyapatite nanocomposite rods via in situ hybridization: a potential material as internal fixation of bone fracture. Biomaterials 2004; 25(5):779-85.

[15] Frenkel SR, Bradica G, Brekke JH, Goldman SM, Ieska K, Issack P, et al. Regeneration of articular cartilage-evaluation of osteochondral defect repair in the rabbit using multiphasic implants. Osteoarthritis Cartilage 2005;13: 798-807.

[16] Montembault A, Tahiri K, Korwin-Zmijowska C, Chevalier X, Corvol MT Domard A. A material decoy of biological media based on chitosan physical hydrogels: application to cartilage tissue engineering. Biochimie 2006;88(5): 551-64.

[17] Hoemann CD, Sun J, Legare A, McKee MD, Buschmann MD. Tissue engineering of cartilage using an injectable and adhesive chitosan-based cell-delivery vehicle. Osteoarthritis Cartilage 2005;13(4):318-29.

[18] Correlo VM, Boesel LF, Pinho E, Costa-Pinto AR, Alves da Silva ML, Bhattacharya $\mathrm{M}$, et al. Melt-based compression-molded scaffolds from chitosan-polyester blends and composites: morphology and mechanical properties. J Biomed Mater Res A 2008.

[19] Correlo VM, Pinho ED, Pashkuleva I, Bhattacharya M, Neves NM, Reis RL. Water absorption and degradation characteristics of chitosan-based polyesters and hydroxyapatite composites. Macromol Biosci 2007;7(3):354-63.

[20] Costa-Pinto AR, Salgado AJ, Correlo VM, Sol P, Bhattacharya M, Charbord P, et al. Adhesion, proliferation and osteogenic differentiation of a mouse mesenchymal stem cell line (BMC9) seeded on novel melt based chitosan/ polyester 3D porous scaffolds. Tissue Eng 2008;14(6):1049-57.

[21] Malafaya PB, Reis RL. Bilayered chitosan-based scaffolds for osteochondral tissue engineering: Influence of hydroxyapatite on in vitro cytotoxicity and dynamic bioactivity studies in a specific double-chamber bioreactor. Acta Biomater 2008.

[22] Oliveira JM, Rodrigues MT, Silva SS, Malafaya PB, Gomes ME, Viegas CA, et al. Novel hydroxyapatite/chitosan bilayered scaffold for osteochondral tissue-engineering applications: scaffold design and its performance when seeded with goat bone marrow stromal cells. Biomaterials 2006;27(36): 6123-37.

[23] Oliveira J, Correlo VM, Sol P, Costa-Pinto A, Salgado A, Bhattacharya M, et al. Assessment of the suitability of chitosan/polybutylene succinate scaffolds seeded with mouse mesenchymal progenitor cells for a cartilage tissue engineering approach. Tissue Eng 2008;14(10):1651-61.

[24] Silva SS, Motta A, Rodrigues MT, Pinheiro AF, Gomes ME, Mano JF. Novel genipin-cross-linked chitosan/silk fibroin sponges for cartilage engineering strategies. Biomacromolecules 2008;9(10):2764-74.

[25] Kuo YC, Lin CY. Effect of genipin-crosslinked chitin-chitosan scaffolds with hydroxyapatite modifications on the cultivation of bovine knee chondrocytes. Biotechnol Bioeng 2006;95(1):132-44.

[26] Yamane S, Iwasaki N, Kasahara Y, Harada K, Majima T, Monde K, et al. Effect of pore size on in vitro cartilage formation using chitosan-based hyaluronic acid hybrid polymer fibers. J Biomed Mater Res A 2007;81(3):586-93. 
[27] Mrugala D, Bony C, Neves N, Caillot L, Fabre S, Moukoko D, et al. Phenotypic and functional characterization of ovine mesenchymal stem cells: application to a cartilage defect model. Ann Rheum Dis 2007.

[28] Malafaya PB, Santos TC, van Griensven M, Reis RL. Morphology, mechanical characterization and in vivo neo-vascularization of chitosan particle aggregated scaffolds architectures. Biomaterials 2008;29(29):3914-26.

[29] Crawford A, Dickinson S. Chondrocyte isolation, expansion, and culture in polymer scaffolds. In: Hollander AP, Hatton PV, editors. Methods in molecular biology. Totowa, NJ: Humana Press; 2004. p. 147-57.

[30] Cheung H-Y, Lau K-T, Lu T-P, Hui D. A critical review on polymer-based bioengineered materials for scaffold development. Composites Part B 2007;38:291-300.

[31] Spiteri CG, Pilliar RM, Kandel RA. Substrate porosity enhances chondrocyte attachment, spreading, and cartilage tissue formation in vitro. J Biomed Mater Res A 2006;78(4):676-83.

[32] Al-Munajjed AA, Hien M, Kujat R, Gleeson JP, Hammer J. Influence of pore size on tensile strength, permeability and porosity of hyaluronan-collagen scaffolds. J Mater Sci Mater Med 2008;19(8):2859-64.

[33] Prendergast PJ, Huiskes R, Soballe K. ESB Research Award 1996. Biophysica stimuli on cells during tissue differentiation at implant interfaces. J Biomech 1997;30(6):539-48

[34] Mainil-Varlet P, Rieser F, Grogan S, Mueller W, Saager C, Jakob RP. Articular cartilage repair using a tissue-engineered cartilage-like implant an animal study. Osteoarthritis Cartilage 2001;9(Suppl. A):S6-15.
[35] Gotterbarm T, Richter W, Jung M, Berardi Vilei S, Mainil-Varlet P, Yamashita T, et al. An in vivo study of a growth-factor enhanced, cell free, two-layered collagen-tricalcium phosphate in deep osteochondral defects. Biomaterials 2006;27(18):3387-95.

[36] Bhardwaj T, Pilliar RM, Grynpas MD, Kandel RA. Effect of material geometry on cartilagenous tissue formation in vitro. J Biomed Mater Res 2001;57(2):190-9.

[37] Nehrer S, Breinan HA, Ramappa A, Young G, Shortkroff S, Louie LK, et al. Matrix collagen type and pore size influence behaviour of seeded canine chondrocytes. Biomaterials 1997;18(11):769-76.

[38] Griffon DJ, Sedighi MR, Schaeffer DV, Eurell JA, Johnson AL. Chitosan scaffolds: interconnective pore size and cartilage engineering. Acta Biomater 2006;2(3):313-20.

[39] Lien SM, Ko LY, Huang TJ. Effect of pore size on ECM secretion and cell growth in gelatin scaffold for articular cartilage tissue engineering. Acta Biomater 2009;5(2):670-9.

[40] Freemont AJ, Hoyland J. Lineage plasticity and cell biology of fibrocartilage and hyaline cartilage: its significance in cartilage repair and replacement. Eur J Radiol 2006;57:32-6.

[41] Freyria AM, Cortial D, Ronziere MC, Guerret S, Herbage D. Influence of medium composition, static and stirred conditions on the proliferation of and matrix protein expression of bovine articular chondrocytes cultured in a 3-D collagen scaffold. Biomaterials 2004;25(4):687-97.

[42] Li WJ, Jiang YJ, Tuan RS. Chondrocyte phenotype in engineered fibrous matrix is regulated by fiber size. Tissue Eng 2006;12(7):1775-85. 